\title{
THE THEORY OF OPTIMAL BALANCING OF MECHANISMS
}

\author{
István PAPP,${ }^{1}$ Márton MÁTÉ ${ }^{2}$ \\ Sapientia Hungarian University of Transylvania, Târgu-Mureș, Faculty of Technical and Human Sciences, \\ Department of Mechanical Engineering, Târgu-Mureș, Romania \\ ${ }^{1}$ pappistvan1944@yahoo.com \\ 2mmate@ms.sapientia.ro
}

\begin{abstract}
This paper presents a general theory regarding the balancing of mechanisms. It starts with Stevenson's theory [1], which proves that any mechanism can be dynamically perfectly balanced if there is a pair of counterweights that are eccentrically positioned in three reciprocating perpendicular axes, passing through the mass center of the body. The system of inertial forces are extended in Fourier series where only the first terms, the basic harmonics, are considered. The proposed goal is to stultify the effect of inertial forces and torques. This paper deals with a situation more appropriate to reality. In common cases there doesn't exist the possibility of implementing counterweights in three perpendicular axes. In this situation only the minimization of inertial forces and torques remains as a possible solution. The method developed for this case is presented.
\end{abstract}

Keywords: mechanism, balancing, optimization.

\section{The mathematical model of the total balancing}

E. N. Stevensen Jr. has presented a research report [1], showing a new, generalized method applicable for balancing of any type of mechanism. He proves that any mechanism can be completely balanced through the implementation of six counterweights, located on three reciprocal perpendicular axes passing through the mass center of the body. These axes fit the frame where the forces and torques applied to the body have been computed. In those particular situations where some of the three counterweight-holder axes are missing, total balancing seems to be impossible. For these cases the author shows briefly the minimization of the amplitude of the vibrations in an arbitrary point of the body. Here it must be emphasized that the paper deals with the minimization of the amplitude in only one node. It also proves that if the minimization there comes true, in the other points the amplitude increases.

Despite of the fact that Stevenson's method cannot offer a solution for the minimization of vibration amplitude in each point of the body, it still remains an outstanding work that can be considered a very robust reference, and one which can be also improved.

In the following, the dynamic balancing will be demonstrated on the general mechanical model shown in Figure 1.

The origin of the frame $O X Y Z$ coincides with the mass center of the body. The balancing axis parallel with $O X$ pins the plane $O Y Z$ in the point $\left(0, a_{z}\right.$, $\left.a_{y}\right)$ The balancing counterweights are fixed on this axis at the distances $X=a_{1}$ and $X=a_{2}$. The counterweights belonging to the other two balancing axes are positioned in a similar way. The balancing axis parallel to $O Y$ pins the plane $O X Z$ in $\left(b_{z}\right.$, $0, b_{x}$ ) while the counterweight distances are, $Y=$ $b_{1}$ and $Y=b_{2}$. Finally, the balancing axis parallel to $O Z$ pins $O X Y$ in $\left(c_{y}, c_{x}, 0\right)$ and the counterweight distances are $Z=c_{1}$ and $Z=c_{2}$.

Due to the eccentricity of counterweight's mass centers they produce during the rotation, centrifugal forces which absolute values are computed with the formula

$$
F=m \omega^{2} r
$$


where $m$ is the mass, and $r$ the eccentricity. The notation of the balancing forces will contain two lower indices: the first denotes the axis $(X, Y$ or $Z$ ) while the second the mass - this can be 1 or 2. Thus $F_{x 1}$ and $F_{x 2}$, appear on the balancing axis parallel to $O X, F_{y 1}$ and $F_{y 2}$ to that parallel with $O Y$ while $F_{z 1}$ and $F_{z 2}$ on the balancing axis parallel with $O Z$.

In the following, it becomes necessary to position the mass centers of the counterweights with reference to the fixed frame of the body. Let us define the phase angle the angle closed between the perpendicular from the mass center to the balancing axis and one of the two remaining axes. In this way, the phase angle of the counterweight mass center fixed on the balancing axis $X$ is measured from axis $Y$ and so on, respecting the circular permutation rule.

In order to compute the balancing mass values, it is necessary to find all phase angles of all counterweights and their static moments, with respect to the frame OXYZ related to the body. The instantaneous angular position of the counterweight mass centers shown on Figure 1. can be given using the expression $\omega t+\phi$ where $\phi$ denotes the phase angle.

The Stevenson's method consists in the complete balancing of the first harmonics of all forces and torques. The principle can be transposed to the terms of any order of the Fourier expansion of the involved forces and torques.

The first term of the Fourier expansion is written in the form $A \cos \omega t+B \sin \omega t$ where the amplitudes $A$ and $B$ are completed with indices referring directions, axes forces or torques.

In conformity with D'Alembert's principle, the counterweight's torques and forces must compensate the swinging forces and torques. Writing this condition for all axes, with respect to Figure 1., the following systems of equation result:

$$
\begin{aligned}
& \sum F_{X}=0 ; A_{F X} \cos \omega t+B_{F X} \sin \omega t+ \\
& +F_{Z 1} \cos \left(\omega t+\varphi_{Z 1}\right)+F_{Z 2} \cos \left(\omega t+\varphi_{Z 2}\right)+ \\
& +F_{Y 1} \sin \left(\omega t+\varphi_{Y 1}\right)+F_{Y 2} \sin \left(\omega t+\varphi_{Y 2}\right)=0 \\
& \sum F_{y}=0 ; A_{F Y} \cos \omega t+B_{F Y} \sin \omega t+ \\
& +F_{X 1} \cos \left(\omega t+\varphi_{X 1}\right)+F_{X 2} \cos \left(\omega t+\varphi_{X 2}\right)+ \\
& +F_{Z 1} \sin \left(\omega t+\varphi_{Z 1}\right)+F_{Z 2} \sin \left(\omega t+\varphi_{Z 2}\right)=0 \\
& \sum F_{Z}=0 ; A_{F Z} \cos \omega t+B_{F Z} \sin \omega t+ \\
& +F_{Y 1} \cos \left(\omega t+\varphi_{Y 1}\right)+F_{Y 2} \cos \left(\omega t+\varphi_{Y 2}\right)+ \\
& +F_{X 1} \sin \left(\omega t+\varphi_{X 1}\right)+F_{X 2} \sin \left(\omega t+\varphi_{X 2}\right)=0
\end{aligned}
$$

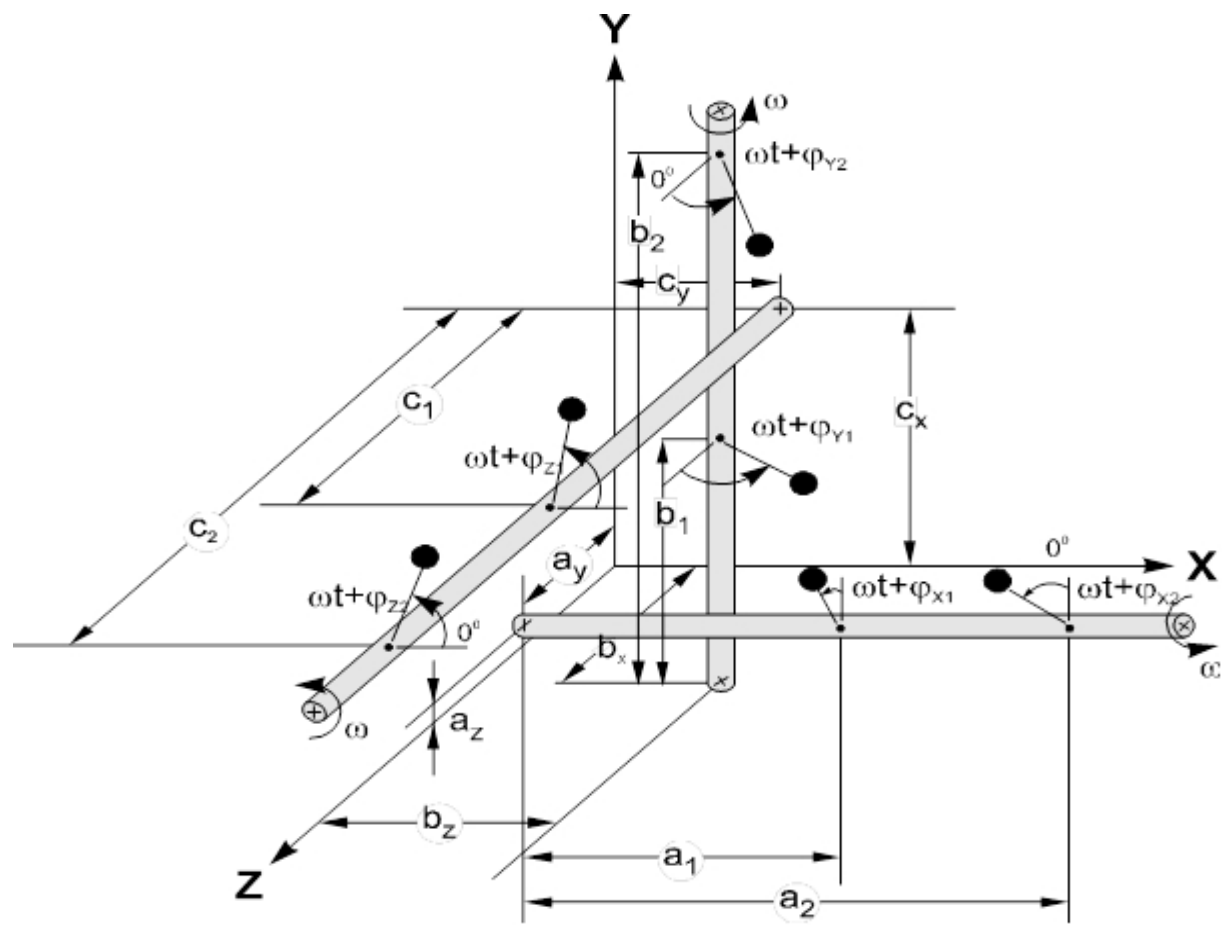

Figure 1. The generalized mechanical model of dynamic balancing 
$\sum M_{x}=0 ;$

$A_{M X} \cos \omega t+B_{M X} \sin \omega t+b_{1} F_{Y 1} \cos \left(\omega t+\varphi_{Y 1}\right)$

$+b_{2} F_{Y 2} \cos \left(\omega t+\varphi_{Y 2}\right)-c_{1} F_{Z 1} \sin \left(\omega t+\varphi_{Z 1}\right)-$

$-c_{2} F_{Z 2} \sin \left(\omega t+\varphi_{Z 2}\right)-a_{y} F_{X 1} \cos \left(\omega t+\varphi_{X 1}\right)-$

$-a_{y} F_{X 2} \cos \left(\omega t+\varphi_{X 2}\right)+a_{z} F_{X 1} \sin \left(\omega t+\varphi_{X 1}\right)+$

$+a_{z} F_{X 2} \sin \left(\omega t+\varphi_{X 2}\right)=0$

$\sum M_{y}=0 ;$

$A_{M Y} \cos \omega t+B_{M Y} \sin \omega t+c_{1} F_{Z 1} \cos \left(\omega t+\varphi_{Z 1}\right)+$

$+c_{2} F_{Z 2} \cos \left(\omega t+\varphi_{Z 2}\right)-a_{1} F_{X 1} \sin \left(\omega t+\varphi_{X 1}\right)-$

$-a_{2} F_{X 2} \sin \left(\omega t+\varphi_{X 2}\right)-b_{Z} F_{Y 1} \cos \left(\omega t+\varphi_{Y 1}\right)-$

$b_{z} F_{Y 2} \cos \left(\omega t+\varphi_{Y 2}\right)+b_{x} F_{Y 1} \sin \left(\omega t+\varphi_{Y 1}\right)+$

$b_{x} F_{Y 2} \sin \left(\omega \cdot t+\varphi_{Y 2}\right)=0$

$\sum M_{Z}=0$;

$A_{M Z} \cos \omega t+B_{M Z} \sin \omega t+a_{1} F_{X 1} \cos \left(\omega t+\varphi_{X 1}\right)+$

$+a_{2} F_{X 2} \cos \left(\omega t+\varphi_{X 2}\right)-b_{1} F_{Y 1} \sin \left(\omega t+\varphi_{Y 1}\right)-$

$-b_{2} F_{Y 2} \sin \left(\omega t+\varphi_{Y 2}\right)+c_{y} F_{Z 1} \sin \left(\omega t+\varphi_{Z 1}\right)+$

$+c_{y} F_{Z 2} \sin \left(\omega t+\varphi_{Z 2}\right)-c_{x} F_{Z 1} \cos \left(\omega t+\varphi_{Z 1}\right)-$

$-c_{x} F_{Z 2} \cos \left(\omega t+\varphi_{Z 2}\right)=0$

(3)

Let' simplify these equations by introducing of the following notations:

$$
\begin{aligned}
Q_{1}=F_{X 1} \cos \varphi_{X 1} & Q_{2}=F_{X 2} \cos \varphi_{X 2} \\
R_{1}=F_{X 1} \sin \varphi_{X 1} & R_{2}=F_{X 2} \sin \varphi_{X 2} \\
S_{1}=F_{Y 1} \cos \varphi_{Y 1} & S_{2}=F_{Y 2} \cos \varphi_{Y 2} \\
T_{1}=F_{Y 1} \sin \varphi_{Y 1} & T_{2}=F_{Y 2} \sin \varphi_{Y 2} \\
U_{1}=F_{Z 1} \cos \varphi_{Z 1} & U_{2}=F_{Z 2} \cos \varphi_{Z 2} \\
V_{1}=F_{Z 1} \sin \varphi_{Z 1} & V_{2}=F_{Z 2} \sin \varphi_{Z 2}
\end{aligned}
$$

Using these, equations (2) and (3) will suit the following shape:

$$
\begin{aligned}
& \sum F_{X}=0 ;\left(A_{F X}+U_{1}+U_{2}+T_{1}+T_{2}\right) \cos \omega t+ \\
&+\left(B_{F X}-V_{1}-V_{2}+S_{1}+S_{2}\right) \sin \omega t=0 \\
& \sum F_{y}=0 ;\left(A_{F Y}+Q_{1}+Q_{2}+V_{1}+V_{2}\right) \cos \omega t+ \\
&+\left(B_{F Y}+U_{1}+U_{2}-R_{1}-R_{2} \sin \omega t=0\right. \\
& \sum F_{z}=0 ;\left(A_{F Z}+S_{1}+S_{2}+R_{1}+R_{2}\right) \cos \omega t+ \\
&+\left(B_{F Z}+Q_{1}+Q_{2}-T_{1}-T_{2}\right) \sin \omega t=0
\end{aligned}
$$

$\sum M_{x}=0 ; E_{X 1} \cos \omega t+E_{X 2} \sin \omega t=0$

$\sum M_{y}=0 ; E_{Y 1} \cos \omega t+E_{Y 2} \sin \omega t=0$

$\sum M_{Z}=0 ; E_{Z 1} \cos \omega t+E_{Z 2} \sin \omega t=0$

$E_{X 1}=\left(A_{M X}+b_{1} S_{1}+b_{2} S_{2}-c_{1} V_{1}-c_{2} V_{2}-\right.$

$$
\left.-a_{y} Q_{1}-a_{y} Q_{2}+a_{z} R_{1}+a_{z} R_{2}\right)
$$

$E_{X 2}=\left(B_{M X}-b_{1} T_{1}-b_{2} T_{2}-c_{1} U_{1}-c_{2} U_{2}+\right.$

$$
\left.+a_{z} Q_{1}+a_{z} Q_{2}+a_{y} R_{1}+a_{y} R_{2}\right)
$$

$$
\begin{aligned}
& E_{Y 1}=\left(A_{M Y}-a_{1} R_{1}-a_{2} R_{2}+c_{1} U_{1}+c_{2} U_{2}-\right. \\
& \left.-b_{z} S_{1}-b_{z} S_{2}+b_{x} T_{1}+b_{x} T_{2}\right) \\
& E_{Y 2}=\left(B_{M Y}-c_{1} V_{1}-c_{2} V_{2}-a_{1} Q_{1}-a_{2} Q_{2}+\right. \\
& \left.+b_{z} T_{1}+b_{z} T_{2}+b_{x} S_{1}+b_{x} S_{2}\right) \\
& E_{Z 1}=\left(A_{M Z}+a_{1} Q_{1}+a_{2} Q_{2}-b_{1} T_{1}-b_{2} T_{2}+\right. \\
& \left.c_{y} V_{1}+c_{y} V_{2}-c_{x} U_{1}-c_{x} U_{2}\right) \\
& E_{Z 2}=\left(B_{M Z}-a_{1} R_{1}-a_{2} R_{2}-b_{1} S_{1}-b_{2} S_{2}+\right. \\
& \left.c_{y} U_{1}+c_{y} U_{2}+c_{x} V_{1}+c_{x} V_{2}\right)
\end{aligned}
$$

The balancing status is achieved only if equations (5) are valid for the whole kinematic cycle, or, in other terms, they must not depend on the time variable $t$. This becomes possible only if terms in cos $\omega t$ and sin $\omega t$ are zero. This condition generates a linear system of 12 equations and 12 unknowns whose matrix form is given in matrix (6).

Using the root of system (6) the absolute values of the balancing forces will be computed using formula (4). Thus results:

$$
\begin{aligned}
& F_{X 1}=\sqrt{Q_{1}^{2}+R_{1}^{2}}=(m r)_{X 1} \omega^{2} \\
& F_{X 2}=\sqrt{Q_{2}^{2}+R_{2}^{2}}=(m r)_{X 2} \omega^{2} \\
& F_{Y 1}=\sqrt{S_{1}^{2}+T_{1}^{2}}=(m r)_{Y 1} \omega^{2} \\
& F_{Y 2}=\sqrt{S_{2}^{2}+T_{2}^{2}}=(m r)_{Y 2} \omega^{2} \\
& F_{Z 1}=\sqrt{U_{1}^{2}+V_{1}^{2}}=(m r)_{Z 1} \omega^{2} \\
& F_{Z 2}=\sqrt{U_{2}^{2}+V_{2}^{2}}=(m r)_{Z 2} \omega^{2}
\end{aligned}
$$

The phase angles are given by the following equations: 


$$
\left[\begin{array}{cccccccccccc}
0 & 0 & 0 & 0 & 0 & 0 & 1 & 1 & 1 & 1 & 0 & 0 \\
0 & 0 & 0 & 0 & 1 & 1 & 0 & 0 & 0 & 0 & -1 & -1 \\
1 & 1 & 0 & 0 & 0 & 0 & 0 & 0 & 0 & 0 & 1 & 1 \\
0 & 0 & -1 & -1 & 0 & 0 & 0 & 0 & 1 & 1 & 0 & 0 \\
0 & 0 & 1 & 1 & 1 & 1 & 0 & 0 & 0 & 0 & 0 & 0 \\
1 & 1 & 0 & 0 & 0 & 0 & -1 & -1 & 0 & 0 & 0 & 0 \\
-a_{y} & -a_{y} & a_{z} & a_{z} & b_{1} & b_{2} & 0 & 0 & 0 & 0 & -c_{1} & -c_{2} \\
a_{z} & a_{z} & a_{y} & a_{y} & 0 & 0 & -b_{1} & -b_{2} & -c_{1} & -c_{2} & 0 & 0 \\
0 & 0 & -a_{1} & -a_{2} & -b_{z} & -b_{z} & b_{x} & b_{x} & c_{1} & c_{2} & 0 & 0 \\
-a_{1} & -a_{2} & 0 & 0 & b_{x} & b_{x} & b_{z} & b_{x} & 0 & 0 & -c_{1} & -c_{2} \\
a_{1} & a_{2} & 0 & 0 & 0 & 0 & -b_{1} & -b_{2} & -c_{x} & -c_{x} & c_{y} & c_{y} \\
0 & 0 & -a_{1} & -a_{2} & -b_{1} & -b_{2} & 0 & 0 & c_{y} & c_{y} & c_{x} & c_{x}
\end{array}\right] \cdot\left[\begin{array}{l}
Q_{1} \\
Q_{2} \\
R_{1} \\
R_{2} \\
S_{1} \\
S_{2} \\
T_{1} \\
T_{2} \\
U_{1} \\
U_{2} \\
V_{1} \\
V_{2}
\end{array}\right]=\left[\begin{array}{l}
-A_{F X} \\
-B_{F X} \\
-A_{F Y} \\
-B_{F Y} \\
-A_{F Z} \\
-B_{F Z} \\
-A_{M X} \\
-B_{M X} \\
-A_{M Y} \\
-B_{M Y} \\
-A_{M Z} \\
-B_{M Z}
\end{array}\right]
$$

$$
\begin{aligned}
\phi_{X i} & =\operatorname{arctg} \frac{R_{i}}{Q_{i}} \\
\phi_{Y i} & =\operatorname{arctg} \frac{T_{i}}{S_{i}} \\
\phi_{Z i} & =\operatorname{arctg} \frac{V_{i}}{U_{i}}, i \in\{1 ; 2\}
\end{aligned}
$$

$$
\begin{aligned}
& \sum F_{x}=A_{\Sigma F_{x}} \cos \omega t+B_{\Sigma F_{x}} \sin \omega t \\
& \sum F_{y}=A_{\Sigma F_{y}} \cos \omega t+B_{\Sigma F_{y}} \sin \omega t \\
& \sum F_{z}=A_{\Sigma F_{z}} \cos \omega t+B_{\Sigma F_{z}} \sin \omega t
\end{aligned}
$$

The coefficients $A_{\Sigma F_{k}}, B_{\Sigma F_{k}}, k \in\{X ; Y ; Z\}$ of system (9) present a formal identity with the coefficients of terms and from system (5). Here it must be emphasized that the expressions in parentheses are built up with the coefficients of the inertial forces and torques like $A_{F k}, B_{F K} A_{M k}, B_{M k}$ $k \in\{X ; Y ; Z\}$ from the equations (5) and from the coefficients of the balancing torques and forces. Putting together the systems of equations (5) and (9), the equality of the coefficients of the equivalent terms can be written as follows:

\section{Optimal balancing by minimizing of the unbalanced forces and torques acting on the body}

If the balancing can be only partially achieved, the unbalanced forces and torques will produce vibrations that extend to all the system. Due to this, the mechanism becomes noisy. The goal of the optimization consists in the minimizing of the unbalanced forces and torques that act on the body. By applying this method, the values of the unbalanced forces and torques will be kept at their minimal possible values during a complete cycle.

Let us denote the unbalanced forces with $\sum F_{X}, \sum F_{Y}, \sum F_{Z}$ and the unbalanced torques with $\sum M_{X}, \sum M_{Y}, \sum M_{Z}$. In the next step all these time-functions are expanded in Fourier series. Here only the first terms are considered. It results in:

$$
\begin{aligned}
A_{\Sigma F_{X}}= & A_{F X}+U_{1}+U_{2}+T_{1}+T_{2} \\
B_{\Sigma F_{X}}= & B_{F X}-V_{1}-V_{2}+S_{1}+S_{2} \\
A_{\Sigma F_{Y}}= & A_{F Y}+Q_{1}+Q_{2}+V_{1}+V_{2} \\
B_{\Sigma F_{Y}}= & B_{F Y}+U_{1}+U_{2}-R_{1}-R_{2} \\
A_{\Sigma F_{Z}}= & A_{F Z}+S_{1}+S_{2}+R_{1}+R_{2} \\
B_{\Sigma F_{Z}}= & B_{F Z}+Q_{1}+Q_{2}-T_{1}-T_{2} \\
A_{\Sigma M_{X}}= & A_{M X}+b_{1} S_{1}+b_{2} S_{2}-c_{1} V_{1}-c_{2} V_{2}- \\
& -a_{y}\left(Q_{1}+Q_{2}\right)+a_{z}\left(R_{1}+R_{2}\right) \\
B_{\Sigma M_{X}}= & B_{M X}-b_{1} T_{1}-b_{2} T_{2}-c_{1} U_{1}-c_{2} U_{2}+ \\
& +a_{z}\left(Q_{1}+Q_{2}\right)+a_{y}\left(R_{1}+R_{2}\right)
\end{aligned}
$$




$$
\begin{aligned}
A_{\Sigma M_{Y}}= & A_{M Y}-a_{1} R_{1}-a_{2} R_{2}+c_{1} U_{1}+c_{2} U_{2}- \\
& -b_{z}\left(S_{1}+S_{2}\right)+b_{x}\left(T_{1}+T_{2}\right) \\
B_{\Sigma M_{Y}}= & B_{M Y}-c_{1} V_{1}-c_{2} V_{2}-a_{1} Q_{1}-a_{2} Q_{2}+ \\
& +b_{z}\left(T_{1}+T_{2}\right)+b_{x}\left(S_{1}+S_{2}\right) \\
A_{\Sigma M_{Z}}= & A_{M Z}+a_{1} Q_{1}+a_{2} Q_{2}-b_{1} T_{1}-b_{2} T_{2}+ \\
& +c_{y}\left(V_{1}+V_{2}\right)-c_{x}\left(U_{1}+U_{2}\right) \\
B_{\Sigma M_{Z}}= & B_{M Z}-a_{1} R_{1}-a_{2} R_{2}-b_{1} S_{1}-b_{2} S_{2}+ \\
& +c_{y}\left(U_{1}+U_{2}\right)+c_{x}\left(V_{1}+V_{2}\right)
\end{aligned}
$$

The Fourier- coefficients of the unbalanced inertial forces and torques are primed by the following matrix-equation

$$
\mathbf{R}_{(i)}=\mathbf{P}_{(i j)} \mathbf{Q}_{(j)}+\mathbf{F}_{(i)}
$$

The terms of the equation above have the fol-lowing meanings:

$-\mathbf{R}_{(i)}$ a vertex of $i$ elements containing the Fourier coefficients of the residual unbalanced forces and torques that persist after optimization;

$-\mathbf{P}_{(i j)}$ a matrix of $i$ rows and $j$ columns that contains the position data of the balancing forces and torques;

$-\mathbf{Q}_{(j)}$ the vertex of $j$ elements of the optimal balancing forces;

$-\mathbf{F}_{(i)}$ the vertex of $i$ elements containing the Fourier coefficients of the inertial forces and torques. In the next the indices referring to the number of rows and columns are omitted.

The matrix equation suits particular forms depending on the number of the balancing axes. In the case of 2 balancing axes $i=1,2, \ldots, 12$ and $j=1, \ldots$, 8. If there exists only one balancing axis then $i=1$, $2, \ldots, 10$ and $j=1, \ldots, 4$.

In order to perform the minimizing of the unbalanced forces and torques the quadratic form of the coefficient-function will be used. It can be written in the following form:

$$
U=\mathbf{R}^{T} \mathbf{R}
$$

The optimizing consists in finding the minimum of (12). For this it is necessary to compute the $\mathbf{Q}^{-}$derivative of the quadratic form $\mathrm{U}$, namely the sum of partial derivatives by any $Q_{k}$-term:

$$
\begin{aligned}
& \frac{\mathrm{d} U}{\mathrm{~d} \mathbf{Q}}=\sum_{k=1}^{j} \frac{\partial U}{\partial Q_{k}}= \\
& =\sum_{k=1}^{j}\left(\left[\frac{\partial \mathbf{R}}{\partial Q_{k}}\right]^{T} \mathbf{R}+\mathbf{R}^{T} \frac{\partial \mathbf{R}}{\partial Q_{k}}\right)
\end{aligned}
$$

Let us observe that vertex $\mathbf{R}$ and its partial derivatives meet the following condition

$$
\left[\frac{\partial \mathbf{R}}{\partial Q_{k}}\right]^{T} \mathbf{R}=\mathbf{R}^{T} \frac{\partial \mathbf{R}}{\partial Q_{k}}
$$

Applying this, equation (13) turns into a more advantageous form:

$$
\frac{\mathrm{d} U}{\mathrm{~d} \mathbf{Q}}=2 \sum_{k=1}^{j}\left(\left[\frac{\partial \mathbf{R}}{\partial Q_{k}}\right]^{T} \mathbf{R}\right)=2 \sum_{k=1}^{j}\left(\mathbf{R}^{T} \frac{\partial \mathbf{R}}{\partial Q_{k}}\right)
$$

Using the form (11) of vertex $\mathbf{R}$, the partial derivatives of $Q_{k}, k=\overline{1 . . j}$ can be primed as follows:

$$
\frac{\partial \mathbf{R}}{\partial Q_{k}}=\frac{\partial}{\partial Q_{k}}(\mathbf{P Q}+\mathbf{F})
$$

Due to the fact that vertices $\mathbf{P}$ and $\mathbf{F}$ are not depending on the $Q_{k}, k=\overline{1 . . j}$ balancing forces, the partial derivatives are zeros:

$$
\frac{\partial \mathbf{P}}{\partial Q_{k}}=0 ; \frac{\partial \mathbf{F}}{\partial Q_{k}}=0
$$

Using this, expression (16) can be written as

$$
\frac{\partial \mathbf{R}}{\partial Q_{k}}=\frac{\partial}{\partial Q_{k}}(\mathbf{P Q})=\mathbf{P} \frac{\partial \mathbf{Q}}{\partial Q_{k}}
$$

On the other hand

$$
\begin{aligned}
{\left[\frac{\partial \mathbf{R}}{\partial Q_{k}}\right]^{T} } & =\left[\frac{\partial}{\partial Q_{k}}(\mathbf{P Q})\right]^{T}= \\
& =\frac{\partial}{\partial Q_{k}}\left(\mathbf{Q}^{T} \mathbf{P}^{T}\right)=\frac{\partial \mathbf{Q}^{T}}{\partial Q_{k}} \mathbf{P}^{T}
\end{aligned}
$$

Substituting expressions (11), (18) and (19) into equation (15), after performing a convenient arrangement of terms, the following form results:

$$
\begin{aligned}
\frac{\mathrm{d} U}{\mathrm{~d} \mathbf{Q}} & =2 \sum_{k=1}^{j}\left(\left[\frac{\partial \mathbf{R}}{\partial Q_{k}}\right]^{T} \mathbf{R}\right)= \\
& =2 \sum_{k=1}^{j}\left(\frac{\partial \mathbf{Q}^{T}}{\partial Q_{k}} \mathbf{P}^{T}(\mathbf{P Q}+\mathbf{F})\right)
\end{aligned}
$$

The local minimum of $U$ is given by:

$$
\frac{\mathrm{d} U}{\mathrm{~d} \mathbf{Q}}=0
$$

Let us emphasize that the $Q_{k}$ derivative of the vertex $Q$ results with all its elements zero, except $\mathrm{k}^{\text {th }}$ 
which equals 1 . After performing the summation in equation (20), the condition (21) becomes:

$$
\mathbf{P}^{T}(\mathbf{P Q}+\mathbf{F})=0
$$

Or in extended form:

$$
\left[\mathbf{P}^{T} \mathbf{P}\right] \mathbf{Q}+\mathbf{P}^{T} \mathbf{F}=0
$$

Let us introduce the following notations:

$$
\begin{aligned}
& \mathbf{N}=\mathbf{P}^{T} \mathbf{P} \\
& \mathbf{K}=\mathbf{P}^{T} \mathbf{F}
\end{aligned}
$$

With this, equation (23) turns into a linear form with respect to $\mathbf{Q}$ :

$$
\mathbf{N Q}+\mathbf{K}=0
$$

Finally the Q-root can be primed as:

$$
\mathbf{Q}=-\mathbf{N}^{-1} \mathbf{K}
$$

\section{Application}

Let's suppose that there exists only one balancing axis superposed with $O Z$, where the counterweights are mounted.

As a consequence, the machine is missing balancing axes parallel to $O X$ and $O Y$ so $F_{X i}=F_{Y i}=0$, The result is that all components of the $\mathbf{F}$ forces are zeros: $Q_{i}=R_{i}=S_{i}=T_{i}=0, \mathrm{i}=1,2$.

As proven in paper [1] the inertial forces and torques cannot be balanced using counter-weights placed on only a single axis. By applying equations (9) and (10) these can by partially decreased.

On the right side of matrix equation (6) we write the Fourier coefficients of the residual unbalanced forces and torques:
While the machine is missing the balancing axes parallel to $O X$ and $O Y$ and the correspondent counterweights, the coordinates of these become unnecessary, and thus will equate to zero. By paying attention to equations (6), (9) and (10), matrix equation (28) can be transformed into the particular form shown below:

$$
\left[\begin{array}{l}
\mathbf{A}_{1} \\
\mathbf{A}_{2}
\end{array}\right]\left[\begin{array}{l}
\mathbf{U} \\
\mathbf{V}
\end{array}\right]+\mathbf{F}=\mathbf{F}_{\Sigma}
$$

Equation (29) uses the following notations:

$$
\mathbf{A}_{1}=\left(\begin{array}{cccc}
1 & 1 & 0 & 0 \\
0 & 0 & -1 & -1 \\
0 & 0 & 1 & 1 \\
0 & 0 & -c_{1} & -c_{2} \\
c_{1} & c_{2} & 0 & 0
\end{array}\right)
$$

$$
\begin{aligned}
& \mathbf{A}_{2}=\left(\begin{array}{cccc}
-c_{1} & -c_{2} & 0 & 0 \\
c_{1} & c_{2} & 0 & 0 \\
0 & 0 & -c_{1} & -c_{2} \\
0 & 0 & -c_{1} & -c_{2} \\
-c_{x} & -c_{x} & c_{y} & c_{y}
\end{array}\right) \\
& \mathbf{U}=\left(\begin{array}{ll}
U_{1} & U_{2}
\end{array}\right)^{T} \\
& \mathbf{V}=\left(\begin{array}{ll}
V_{1} & V_{2}
\end{array}\right)^{T}
\end{aligned}
$$

$$
\begin{array}{r}
\mathbf{F}=\left(\begin{array}{lllll}
A_{F X} & B_{F X} & A_{F Y} & B_{F Y} & A_{M X} \cdots \\
B_{M X} & A_{M Y} & B_{M Y} & A_{M Z} & B_{M Z}
\end{array}\right)^{T}
\end{array}
$$




$$
\begin{array}{rlll}
\mathbf{F}_{\Sigma}= & \left(\begin{array}{llll}
A_{\Sigma F X} & B_{\Sigma F X} & A_{\Sigma F Y} & B_{\Sigma F Y} \cdots \\
A_{\Sigma F Z} & B_{\Sigma F Z} & A_{\Sigma M X} & B_{\Sigma M X} \cdots \\
A_{\Sigma M Y} & B_{\Sigma M Y} & A_{\Sigma F Z} & B A_{\Sigma M Z}
\end{array}\right)^{T}
\end{array}
$$

System (29) consists of 10 equations and contains 14 unknowns. In this case, the problem of balancing should be formulated in the following manner: where and what counterweights must be fixed on the axis of the machine, so that the amplitudes of the residual unbalanced forces and torques decrease to the minimum possible? The answer can be achieved through solving equations (27), (29), (18) and (19).

\section{Conclusions}

The procedure of balancing presented in this paper is in many ways more advantageous in comparison with traditional methods. The writing of equations must be preceded by the geometric mapping of the mechanism (computing of mass centers, principal axes of inertia). This, by using modern modeling software reduces to a common task.

Although the present paper deals only with the first terms of the Fourier expansions, the procedure can be generalized by taking into consideration a finite number of terms. This leads to a significantly larger number of unknowns that requires an increased computing capacity.

\section{References}

[1] Stevensen E. N.: Balancing of Machines. In: ASME, San Francisco, California, 72/52. 1972.

[2] Papp I., Máté M.: Transformarea generală a două sisteme ortogonale. In: Proceedings CDM 2005, ISBN-973-635-513-6. A IV-a conferinţă de dinamica maşinilor, cu participare internaţională, Braşov, 28-30. mai 2005. Vol. II., 319-326.

[3] Papp I.: Optimization of Dynamical Balancing of Mechanisms According to Diminuation of Vibration Amplitude at a Given Point. In: International Conference in Mechanical Engineering, SatuMare, April 28-May 1, 2005, 285-287. 
